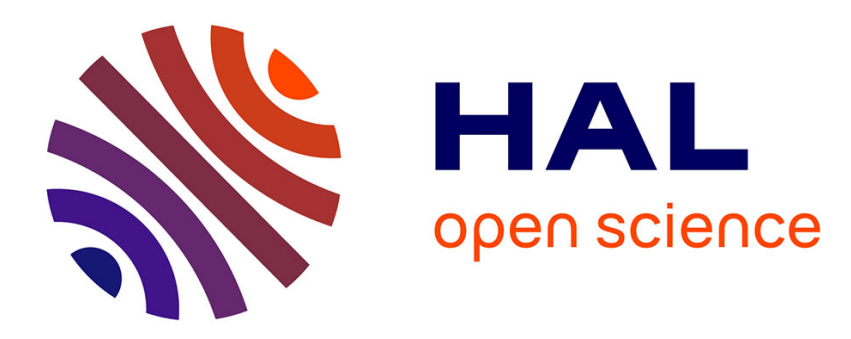

\title{
Les entreprises et le développement durable
}

Franck Aggeri, Olivier Godard

\section{To cite this version:}

Franck Aggeri, Olivier Godard. Les entreprises et le développement durable. Entreprises et Histoire, 2006, 45, pp.6-19. halshs-00645674

\section{HAL Id: halshs-00645674 https://shs.hal.science/halshs-00645674}

Submitted on 1 Dec 2014

HAL is a multi-disciplinary open access archive for the deposit and dissemination of scientific research documents, whether they are published or not. The documents may come from teaching and research institutions in France or abroad, or from public or private research centers.
L'archive ouverte pluridisciplinaire HAL, est destinée au dépôt et à la diffusion de documents scientifiques de niveau recherche, publiés ou non, émanant des établissements d'enseignement et de recherche français ou étrangers, des laboratoires publics ou privés. 


\title{
$\begin{array}{lllllllll}\mathbf{E} & \mathbf{D} & \mathbf{I} & \mathbf{T} & \mathbf{O} & \mathbf{R} & \mathbf{I} & \mathbf{A} & \mathbf{L}\end{array}$ \\ LES ENTREPRISES ET LE DÉVELOPPEMENT DURABLE
}

\author{
par Franck AGGERI \\ Centre de Gestion Scientifique \\ Ecole des Mines \\ et \\ Olivier GODARD \\ Laboratoire d'Économétrie \\ CNRS - Ecole Polytechnique
}

Le développement durable est incontestablement une question d'actualité. Formée historiquement comme projet politique, cette idée du développement a également émergé dans les quinze dernières années comme projet managérial. L'atteste la naissance de pratiques gestionnaires dans les grandes entreprises (discours, communication, outils, etc.), de formes de régulation (normes volontaires et réglementaires en matière de reporting extra-financier) ou, selon l'heureuse expression de David Vogel, de "marchés de la vertu "'". Ces derniers voient prospérer consultants, auditeurs, certificateurs qui évaluent la performance sociétale des entreprises, labellisent des produits « éthiques » et proposent leurs offres de conseils. A cette aune, le projet managérial du développement durable présente deux caractéristiques principales : il concerne potentiellement tous les domaines d'activités de l'entreprise - la stratégie générale, la communication. la gouvernance d'entreprise, la conception de produits, les activités productives, etc. - : il repose sur la promesse d'un capitalisme œuvrant à sa réconciliation avec l'ensemble de la société en faisant siennes les préoccupations de cette dernière comme la responsabilité environnementale et l'équité sociale au sein du processus de développement économique.

\section{LES CONDITIONS D'UNE RECHERCHE HISTORIQUE SUR LES ENTREPRISES ET LE DÉVELOPPEMENT DURABLE}

L'émergence d'une question d'actualité suffit-elle à en faire un thème de recherche sur le plan historique? La notion n'est-elle pas trop galvaudée, saturée de discours et d'interpréta-

(1) Voir D. Vogel. The market for virtue. The potental and limits of corporate social responsibility, Washington, Brookings Institution Press. 2005. 
tions pour permettre la prise de recul nécessaire ? Ne s'agit-il pas d'un nouvel avatar des modes managériales, voué à disparaître aussi vite qu'il est apparu ? Les lecteurs de la revue peuvent légitimement se poser ces questions. Ils trouveront peu d'éléments de réponse historiques et empiriques à ces questions dans l'abondante littérature managériale et dans la presse qui se fait l'écho de ces débats. Les sceptiques trouveront matière à renforcer leur conviction dans la critique. Cette dernière réunit, de façon inattendue, les défenseurs de la création de valeur pour l'actionnaire et les pourfendeurs de la grande entreprise, pour qui il deviendra tôt ou tard évident que les objectifs de recherche du profit sont inconciliables avec un développement durable, mais aussi les défenseurs de la société civile, pour qui il n'est pas du ressort de l'entreprise de prendre en charge des questions sociétales qui relèvent de l'action collective, puis publique. Les plus optimistes, qui pensent qu'une transformation profonde du capitalisme est en cours, trouveront des arguments dans un courant de littérature qui donne à montrer les exemples de bonnes pratiques de gestion des relations avec les parties prenantes. Ils n'en trouveront pas beaucoup plus dans l'importante littérature en sciences sociales ${ }^{(2)}$ qui se fonde, pour l'essentiel, sur une conception essentialisée des rapports entre l'entreprise et la société - souvent résumée à une liste de parties prenantes dont l'identité et les intérêts sont connus. Les questions sont alors : comment l'entreprise doit-elle gérer les relations avec les parties prenantes pour légitimer son action ou améliorer son efficacité ? Comment mesurer la performance sociale de l'entreprise?

Posé dans des termes aussi universels et ahistoriques, le débat sur la responsabilité de l'entreprise et sa contribution aux objectifs de développement durable est une question indécidable. Est-il possible, en effet, de trancher un tel débat indépendamment des contextes institutionnels et sociaux et des époques dans lesquels il s'inscrit ? Dans quels termes poser des questions de recherche adaptées à une démarche historique ? Trois éléments de méthode nous semblent utiles à préciser.

Premier élément : laisser de côté la question de la nature de l'entreprise et de ses parties prenantes n'implique pas de tomber dans un empirisme débridé. Elle consiste, sur le plan théorique, à considérer l'entreprise, pour reprendre la proposition de Armand Hatchuel, « comme un collectif qui n'est pas comme les autres : elle a pour particularité d'être éphémère et de n'admettre aucune définition naturelle $[. .$.$] et la révision de ses frontières (physiques, légales,$ humaines, commerciales, etc.) est une condition de son existence ${ }^{(33}$. Si l'on accepte cette caractérisation, la question des projets de l'entreprise et de leurs transformations historiques constitue un premier champ d'investigation pour la recherche. Projets qui impliquent des engagements, des processus, des formes d'action collective, des outils et des démarches dont il convient alors d'étudier les formes et les rationalisations successives.

Deuxième élément : s'intéresser à l'action des entreprises n'implique aucunement de considérer l'entreprise comme une sphère autonome, indépendante des régulations qui s'exer-

(2) Outre les centaines d'ouvrages et d'articles qui traitent du sujet, la communauté scientifique de recherche en gestion est structurée dans le monde anglo-saxon autour de différentes sociétés savantes. Ainsi, il existe depuis 1994 une division de l'Academy of Management (Social Issues of Management) qui traite des rapports de l'entreprise et de la société ainsi qu'une association internationale (I'International Association of Business and Society). En France. outre les manifestations ponctuelles, il existe deux réseaux de recherche permanents : l'Aderse (association pour le développement de la responsabilité sociale des entreprises) et le RIODD (réseau international sur les organisations et le développement durable), rassemblant des chercheurs de différentes disciplines.

(3) Voir A. Hatchuel, "Quel horizon pour les sciences de gestion ? Vers une théorie de l'action collective ", in A. David, A. Hatchuel et R. Laufer (dir.), Les nouvelles fondations des sciences de gestion, Paris, Vuibert, 2000, p. 7-34. 
ceraient sur elle. S'agissant de développement durable, on ne peut ignorer les nouvelles pressions (réglementaires, sociétales et économiques) auxquelles les entreprises sont exposées. Considérer l'activité des entreprises et les formes de régulation collective comme deux champs séparés serait une erreur à la fois sur le plan historique et théorique. D'une part, il faut relativiser l'effet de ces régulations (obligations de reporting sociétal, réglementations environnementales, contestation sociale par des groupes concernés) dont certaines sont plus incitatives que contraignantes. D'autre part, ce serait oublier que les grandes entreprises ont historiquement contribué activement, par leurs activités de lobbying, de partenariat et de mise en exergue de leurs bonnes pratiques, à la production de normes sociales et environnementales, et qu'elles sont désormais officiellement associées à la production de normes mondiales au nom d'une gouvernance mondiale fondée sur une raison partenariale. Si l'on accepte cette deuxième remarque, alors un second champ d'investigation pour la recherche vise à étudier l'évolution des formes de régulation croisées. c'est-à-dire des manières dont les régulations étatiques et sociétales stimulent, orientent et guident l'action des entreprises ; et comment celles-ci participent en retour à la construction de normes sociales et de standards.

Troisième élément : l'omniprésence des discours et de la communication sur le développement durable n'implique pas non plus qu'il faille en conclure à la grande importance empirique de ces formes de pratiques managériales. Certes, elles ont connu un développement et une professionnalisation spectaculaires grâce au concours de consultants qui ont permis, par l'échange des bonnes pratiques, d'aboutir à une certaine uniformisation du langage utilisé par les grandes entreprises mondialisées. Ces discours sont formatés par toute une série d'outils et de technologies, plus ou moins invisibles, qui orientent et transforment ces pratiques discursives. Ceci constitue indéniablement une dimension importante des stratégies de gestion de la contestabilitét ${ }^{+1}$ mises en place par les grandes entreprises industrielles pour anticiper les risques de controverses pouvant toucher à leur réputation et dégrader la valeur des actifs spécifiques dans lesquels elles ont investi. Il y a cependant un risque de myopie à ne s'intéresser qu'aux discours publics de ces grandes entreprises qui, amorcés au début des années 1990 , n'apparaissent de façon massive qu'au début des années 2000. La gestion de la contestabilité ne s'opère pas uniquement par des discours. Elle implique des stratégies complexes se déployant à différents niveaux de l'entreprise. Il convient alors de dépasser une analyse des discours publics pour s'intéresser aux actions et aux changements des technologies managériales. A cette aune, si les discours ont une certaine importance d'un point de vue managérial, c'est moins pour leur valeur intrinsèque que pour leur dimension performative, c'est-à-dire leur capacité à transformer les possibilités d'action et en particulier à rendre possible la valorisation de pratiques locales parfois anciennes dans le domaine social et environnemental. Ces dernières peuvent trouver là l'occasion de réévaluations et de développements inattendus. En retour, pratiques locales et projets d'innovation au sein des entreprises sont susceptibles de nourrir les discours stratégiques, comme l'indiquent certaines recherches contemporaines dans des entreprises pionnières ${ }^{(5)}$. Dans cette perspective, l'analyse historique des pratiques locales de responsabilité sociale sur les sites de production. avec les sous-traitants ou en matière de conception de produits respectueux de l'environnement, est essentielle pour comprendre les conditions d'apprentissages collectifs au-delà des exigences minimales de com-

(4) Voir O. Godard et T. Hommel. «Les multinationales, un enjeu stratégique pour l'environnement et le développement durable? ", La Revue internationale et stratégique, $\mathrm{n}^{\circ}$ 60, hiver 2005-2006, p. 100)-111.

(5) Voir F. Aggeri, É. Pezet. C. Abrassart et A. Acquier, Organiser le développement durable. Expériences des entreprises et formations de règles d'action collective, Paris, Vuibert-Ademe, 2005. 
munication et reporting en matière de développement durable auxquelles toutes les entreprises doivent désormais se conformer. Le seul registre de la gestion des risques de différentes sortes de l'entreprise a peu de chances de produire de tels apprentissages. L'étude des entreprises pionnières doit permettre d'explorer les modalités de développement d'innovations en fonction de nouveaux potentiels de valeur sous-jacents aux nouveaux enjeux de société.

Ainsi, un agenda de recherche historique sur le développement durable dans les entreprises comprend-il trois volets principaux : une étude des évolutions des contours et des projets d'entreprises et de leurs rationalisations, une étude des processus de régulation conjointe entre entreprises et société et une analyse des démarches d'innovation mises en auvre à différents niveaux d'analyse.

\section{2. ÉLÉMENTS D’UNE GÉNÉALOGIE DES CONCEPTS ET DES PRATIQUES}

Avant d'introduire les articles présentés dans ce numéro, il convient de préciser l'origine historique de deux notions qui sont les références omniprésentes des discours d'entreprise et des travaux de recherche dans ce champ : le développement durable et la responsabilité sociale de l'entreprise (RSE). En France, ces deux notions sont souvent utilisées de façon interchangeable tant par les chercheurs que par les acteurs économiques. Elles relèvent pourtant de généalogies et de problématisations différentes que nous allons brièvement rappeler ${ }^{(t)}$.

\section{Le projet politique du développement durable}

Le développement durable a une origine historique précise. L'expression développement durable, traduction de l'anglais sustainable development, a été introduite dans le cercle onusien par l'Union internationale pour la conservation de la nature en 1980 à l'occasion de son rapport proposant une stratégie mondiale pour la conservation de la nature. Elle a ensuite été popularisée à partir de 1988 par le rapport de la Commission mondiale sur l'environnement et le développement, généralement désigné par le nom de sa présidente, Madame Gro Harlem Brundtland, qui a été Premier ministre de son pays, la Norvège. Cette expression prenait le relais d'autres formules proches, comme le développement intégré et surtout, à la suite de la première conférence des Nations unies sur l'environnement tenue à Stockholm en 1972, l'écodéveloppement. La date n'est évidemment pas anodine : la fin des années 1960 et le début des années 1970 correspondent en effet à la réémergence de la question environnementale en termes scientifiques et politiques. Il ne s'agit plus seulement de traiter de « pollutions isolées et locales", mais d'une série de problèmes d'environnement de niveau « régional ». comme les pluies acides, et planétaire (épuisement des ressources naturelles,

(6) Pour une généalogie du projet politique du développement durable et de son insertion dans les débats économiques, on pourra consulter $O$. Godard, "Le développement durable : paysage intellectuel ", Nantres. Sciences et Sociétés, 2 (4), octobre 1994, p. 309-322. Pour une analyse généalogique de la notion de responsabilité sociale des entreprises, on pourra consulter A. Acquier et F. Aggeri, "La responsabilité sociale des entreprises : une revue de la littérature généalogique ", Revue française de gestion, numéro spécial sur la RSE (à paraître), et J. Pasquero, "La responsabilité sociale de l'entreprise comme objet des sciences de gestion : un regard historique ", in M.-F. Turcotte et A. Salmon (dir.), Responsabilité sociale et emironnementale de l'entreprise. Sainte-Foy. Presses universitaires du Québec, 2005. 
perturbations des régulations et des fonctionnements écologiques, enjeux climatiques) qui conduisent à mettre en cause les modes de développement technique et économique prévalents jusqu'alors. De plus, les interdépendances spatiales et temporelles mises en évidence appelaient des formes de gouvernance nouvelles dépassant les frontières politiques des Etatsnations et articulant étroitement politiques locales et régulations globales.

Une caractérisation du développement durable a été retenue du rapport Brundtland : satisfaire les besoins du présent sans compromettre la capacité des générations futures de répondre aux leurs. L'expression a notamment été reprise en France par l'article LII0-1 du Code de l'environnement qui y a ajouté la santé. Le rapport Brundtland précisait que ce développement devait viser en priorité la satisfaction des besoins essentiels des plus démunis et reconnaître les limitations de la capacité de l'environnement à répondre aux besoins. Le texte précisait : «Même au sens le plus étroit du terme, le développement soutenable présuppose un souci d'équité sociale entre les générations, souci qui doit s'étendre, en toute logique. à l'intérieur d'une même génération " $^{77}$.

Le projet politique du développement durable atteste une inflexion par rapport à la notion d'écodéveloppement, davantage centrée sur les enjeux de développement local dans les pays en développement, sur le contenu des techniques et sur les modalités institutionnelles de l'action collective et publique. Formulée en termes plus généraux, la visée du développement durable se présente comme plus œcuménique tout en affirmant la nécessité d'harmoniser les trois références centrales que sont la préservation de l'environnement, le progrès social et le développement économique, comme l’indique la Charte de l'environnement adossée à la Constitution française depuis le $1^{\text {"r }}$ mars $2005^{\left.\right|^{*} \text {. }}$.

Contrairement à une idée reçue qui tend à considérer le développement durable comme un concept flou permettant tous les compromis, l'origine de l'expression est ainsi dénuée d'ambiguïté dans le contexte politique dans lequel elle a été formée. Elle a pour objet de placer les enjeux de la conservation de la nature et de la gestion durable des ressources naturelles au sein d'un projet de développement pour l'Humanité. Loin d'éroder le souci pour la question environnementale, l'idée est qu'il faut transformer les modes de développement déployés depuis le début de l'ère industrielle dans l'ensemble de leurs composantes. Il s’agit, simultanément, de tenir compte des limites environnementales, de donner la priorité aux besoins des plus démunis et d'exercer une responsabilité envers les générations futures, qui seront affectées par les actions des générations présentes à travers l'environnement dont elles hériteront. Sont en cause les structures matérielles des sociétés (consommation, usages du temps, techniques de production, organisation de l'espace) et les modes de régulation qui les pilotent.

Avec le temps, la visée initiale a été lestée d'une approche critique des modes de régulation et d'action publique dans le cadre des Etats-nations, allant jusqu'à reconsidérer la manière de comprendre l'exigence démocratique. D’un côté, les Etats-nations butent sur des limites évidentes d’efficacité et de légitimité pour traiter de questions de portée planétaire. De l'autre côté, la thématique du développement durable a été associée à l'idée d’un réajustement des rapports entre acteurs publics, entreprises et société civile afin de promouvoir une gou-

(7) Commission mondiale sur l'environnement el le développement, Notre avenir à fous, Montréal, Editions du Fleuve, 1988 .

(8) L'article 6 de la Charte stipule : « Les politiques publiques doivent promouvoir un développement durable. A cet effet. elles concilient la protection et la mise en valeur de l'environnement. le développement économique et le progrès social ". 
vernance dépassant les conceptions hiérarchiques classiques du gouvernement représentatif. Cela a conduit différents auteurs à préconiser non seulement la diversification des instruments utilisés, les instruments économiques et volontaires prenant place au côté des approches réglementaires et financières classiques, mais plus fondamentalement à vouloir développer de nouvelles relations de partenariats. Ces dernières visent à développer des coopérations entre instances situées à différents niveaux d'intervention, afin de mieux articuler actions locales et objectifs globaux, et et surtout à associer tous les acteurs sociaux : organisations internationales, administrations publiques, collectivités locales, syndicats, entreprises, consommateurs, chercheurs à un projet de gouvernance mondiale .

Une telle problématique pose naturellement le problème de savoir quel rôle ont à tenir les divers acteurs. Ils sont tous concernés, mais dans quelle mesure et sous quelles formes ? Et pour faire quoi dans quel champ ? La question se pose particulièrement pour les entreprises. Dans leur diversité de taille et d'activité, elles sont des acteurs essentiels de la vie économique. Faut-il considérer pour autant qu'elles sont en première ligne pour promouvoir un développement durable ? Elles organisent la production et développent et choisissent les techniques nouvelles et les produits proposés aux consommateurs. Elles déploient des stratégies commerciales pour convaincre ces consommateurs du besoin qu'ils ont de leurs produits et services. Elles transforment les milieux et déterminent l'usage des ressources. Leurs activités ou leurs produits sont fréquemment sources de pollution, parfois dans des proportions importantes. A contrario, elles sont également capables de réduire, par des actions mobilisant de nouvelles technologies ou de nouveaux produits, ces impacts environnementaux dans des proportions tout aussi considérables. En tant que groupes d'intérêts organisés, à travers fédérations professionnelles et organisations patronales, elles sont aussi des acteurs politiques, prenant part au débat public et exerçant systématiquement un lobbying sur les instances législatives et les responsables des actions publiques. Pour le meilleur et pour le pire, elles sont donc des acteurs dont le comportement et les choix importent pour le développement durable.

La place des entreprises dans le projet politique du développement durable est donc profondément ambivalente : d'un côté, elles ont fortement contribué à la crise de l'environnement, ce que, dans les années 1970, on appelait "les dégâts du progrès "; de l'autre, les grandes entreprises sont devenues des acteurs incontournables, des institutions majeures, qui non seulement participent de facto aux grandes régulations mondiales mais peuvent, si elles y sont incitées et y trouvent un intérêt à long terme, contribuer au projet du développement durable par leurs actions en matière de création de normes sociales, de diffusion des bonnes pratiques environnementales, de développement de nouvelles technologies.

\section{Le projet managérial du développement durable}

Le projet managérial du développement durable se concrétise depuis une dizaine d années à travers une série d'engagements de grandes entreprises et des initiatives concertées volontaires permettant d'organiser le débat entre réseaux d'entreprises, acteurs publics nationaux et organismes internationaux. Deux initiatives en résument la philosophie : le WBCSD et le Pacte Mondial (Global Compact). A l'approche de la Conférence des Nations Unies sur l'Environnement et le Développement qui s'est tenue à Rio de Janeiro en juin 1992, vingt ans après celle de Stockholm, un certain nombre d'entreprises multinationales ont rejoint un club lancé par un entrepreneur suisse, le World Business Council for Sustainable Development. Ce club s'est donné une Charte de bon comportement environnemental et se présente comme le leader du monde des affaires pour la promotion du développement durable. En une quinzaine 
d’années le club s’est élargi jusqu’à rassembler 180 grandes entreprises. Instauré de façon plus récente, en 2001, sous l'égide des Nations Unies, le Pacte Mondial est un code de conduite comportant un ensemble de principes couvrant les droits de l'homme et du travail, la lutte contre la corruption et la responsabilité environnementale. Il a été adopté de manière volontaire par plus de 1400 grandes entreprises.

L'analyse du projet managérial du développement durable permet cependant de mettre en évidence une différence importante par rapport au projet politique du développement durable : aux objectifs environnementaux et d'équité intergénérationnelle propres à ce projet politique le projet managérial ajoute une référence à la Responsabilité Sociale de l’Entreprise, concept provenant du monde anglo-saxon et issu d'une autre généalogie ${ }^{(1) !}$.

\section{La RSE : une histoire nord-américaine}

La notion de RSE est une traduction du terme anglo-saxon de Corporate Social Responsibility (CSR). Selon la définition de la Commission européenne dans son livre vert de 2001 , la RSE fait référence à l'intégration volontaire des préoccupations sociales et écologiques des entreprises à leurs activités commerciales et à leurs relations avec différentes parties concernées (stakeholders) ${ }^{\prime(1) !}$. Alors que le débat sur la RSE peut sembler relativement nouveau en Europe, la littérature économique et gestionnaire nord-américaine témoigne de l'existence et de la vigueur de longue date d'un tel débat aux Etats-Unis, où cette question a historiquement émergé autour de l'éthique religieuse des dirigeants. Le débat s'est structuré sous une forme plus formalisée au début du $X X^{c}$ siècle, à mesure que se sont généralisés le modèle de la grande entreprise à actionnariat dispersé et la figure du dirigeant salarié nonpropriétaire. A partir des années 1920, plusieurs dirigeants se sont exprimés publiquement sur leur responsabilité à l'égard de la société. Si « aucune doctrine clairement formulée de la responsabilité sociale n'avait émergé à la fin de la décennie ", les discours de l'époque étaient très marqués par les concepts de public service et de trusteeship " qui stipulent l'idée d'un contrat implicite, caractérisant la relation entre l'entreprise et la société " "'1".

Pour de nombreux auteurs, l'ouvrage théorique fondateur sur la question est celui de l'économiste Howard R. Bowen : Social responsibilities of the businessman"121. Commandité par une organisation religieuse, le "département de l'église et de la vie économique ", ce livre fournit tout d'abord un témoignage historique très documenté, recense les discours des dirigeants sur la Responsabilité Sociale, entendue comme un ensemble d'obligations à l'égard de la société. Dans son ouvrage. Bowen discute la « doctrine de la responsabilité sociale » (p. 6), alors en vogue dans les milieux d'affaires, et qui stipule qu une approche basée sur l'engagement volontaire des hommes d'affaires pourrait suffire à résoudre les problèmes économiques et sociaux des Etats-Unis d’après-guerre ${ }^{(1.3)}$. Plutôt que d'accepter sans réserve cette

(9) Il est d’ailleurs révélateur de noter que la notion de développement durable (susfaimability) est plus volontiers utilisée par les grandes entreprises européennes, alors que les entreprises américaines font référence à la notion de RSE. (10) Nous avons choisi, dans cet article, de rendre l'anglais stakeholder par "partie concernée ". Le présent alinéa et les trois suivants s`appuient sur A. Acquier et F. Aggeri. "La responsabilité sociale des entreprises... », arr. cit.

(11) Voir M. Heald. The social responsibilities of business: company and community. 1900-1960, Cleveland, Case Western Reserve University Press. 1970.

(12) H. Bowen, Social responsibilities of the businessman. New York. Harper and Brothers, 1953.

(13) Cf. K. Phillips-Fein, "Retour de flamme contre le New Deal : le patronat conservateur et la droite modeme aux Etats-Unis ", Le Mouvement Social, octobre-decembre 2006, p. 73-81. 
proposition, Bowen s'interroge sur les conditions qui permettraient de faire de la Responsabilité Sociale un outil de régulation effectif de l'économie américaine. Il souligne, entre autres, la nécessité de développer de nouveaux outils de gestion tels que l'audit social, ou la nécessité de réformes politiques (développer de nouvelles instances de négociation entre les entreprises et les parties concernées), de transformation des enseignements de gestion, etc. En trame de fond, Bowen développe ainsi une grille d'analyse de l'institutionnalisation de la responsabilité sociale ${ }^{(1+1)}$.

La notion de RSE va connaître un développement important dans les années 1960-1970, dans le contexte d'une mutation profonde de la société américaine (lutte pour les droits des minorités, développement de contre-cultures...), où la responsabilité des entreprises est interpellée à l'occasion de différentes crises : accidents industriels, crises environnementales, discrimination, etc. A cette époque, deux conceptions de la responsabilité de l'entreprise s’opposent. Pour les économistes néo-libéraux, comme Milton Friedman, la notion de RSE est subversive ; la seule responsabilité de l'entreprise est de faire des profits, conduisant à la maximisation du bien-être collectif. A cette responsabilité réduite aux acquêts s'oppose une conception élargie qui met en avant la responsabilité non seulement économique ou juridique mais également morale de l'entreprise par rapport aux conséquences de ses actions ${ }^{151}$. Toutefois cette approche demeure vague et peu opérationnelle pour les managers : vis-à-vis de qui et de quoi cette responsabilité doit-elle s'exercer? La notion va gagner en précision avec la formalisation progressive de la notion de stakeholder (les «parties concernées ») qui va permettre, selon la formule de Carroll, de mettre « des noms et des visages » en face de l'idée de responsabilité. «La notion de stakeholder est un jeu de mots par rapport à la notion de stockholder (les actionnaires) et désigne les individus ou les groupes d'individus qui ont un enjeu, une requête ou un intérêt dans les activités et les décisions de l'entreprise " (Carroll, 1991). Parmi ces stakeholders, on trouve non seulement les partenaires classiques de l'entreprise (actionnaires, clients, salariés), mais plus largement les associations, les ONG, les riverains, les élus locaux, les institutions internationales, etc.) avec qui l'entreprise est amenée à dialoguer, à coopérer ou à développer de nouvelles relations commerciales. Ces notions vont faire l'objet de débats et d'approfondissements théoriques de la part des chercheurs en gestion qui vont notamment s'interroger sur la définition et la qualification des stakeholders ${ }^{\prime(t)}$, sur leur légitimité et leur capacité d'influence ${ }^{177}$, et sur la façon d'analyser et de mesurer la performance sociale de l'entreprise ${ }^{(1 k)}$. Cette approche anglo-saxonne est pragmatique : la responsabilité de l'entreprise n'est pas universelle ; elle est contingente et relative et s'exerce en fonction des stakeholders considérés par l'entreprise comme légitimes. Un moment éclipsée par la vague du modèle actionnarial, cette approche a fait un retour en force depuis la fin des années 1990.

(14) Voir A. Acquier et J.-P. Gond, "Aux sources de la responsabilité sociale de l'entreprise : (re)lecture et analyse d'un ouvrage séminal : Social responsibilities of the businessman d'Howard Bowen (1953) ", communication à la conférence de l'AIMS. Angers. juin 2005.

(15) Voir A.B. Carroll, "The pyramid of corporate social responsibility: toward the moral management of organization stakeholders", Business Horizons, July-August, 1991, p. 39-48.

(16) Voir R.E. Freeman. Strategic management: a stakeholder approach. Marshfield (Mass.). Pitman Publishing. Inc., 1984.

(17) Voir R.K. Mitchell. B.R. Agle and D. Wood, "Toward a theory of stakeholder identification and salience: defining of who and what really counts ". Academy of Management Review, 22(4), 1997, p. 853-886.

(18) Voir D. Wood, "Corporate social performance revisited ". Academy of Management Review', 16(4), 1991. 


\section{L'hybridation entre développement durable et RSE : la " main visible " des prescripteurs}

L'hybridation entre développement durable et RSE s'est opérée dans les années 1990 à travers la « main visible » de consultants britanniques qui ont contribué à l'invention et la diffusion d'un nouveau référentiel universel ${ }^{\mid 1 \text { | }}$. A cette aune, l'entreprise est non seulement responsable à l'égard des communautés locales, des parties concernées actuelles mais également à l'égard de parties concernées incapables de se manifester comme les générations futures. Débarrassé des références à la religion et à l'histoire. le développement durable tend à être présenté comme une stratégie gagnante et donc le produit d'un calcul rationnel des intérêts bien compris. Ainsi, ces consultants ont cherché à mettre en évidence un modèle économique du développement durable (business case) en démontrant qu'il peut être rentable d'être une entreprise durable et socialement responsable ${ }^{2311}$. L'utilisation du terme de développement durable en lien avec les entreprises reflète ainsi la croyance que les objectifs économiques, sociaux et environnementaux ne sont pas foncièrement contradictoires entre eux. L'adoption de démarches de développement durable est d’autant plus nécessaire, expliquent ces prescripteurs, qu'elle s'inscrit dans un mouvement jugé inéluctable d’émergence de nouvelles formes de "régulation civile " $"$ ". Ces dernières ne passent pas par les formes traditionnelles de régulation étatiques, mais se présentent sous la forme de nouvelles demandes de consommateurs pour des produits éthiques, de mouvements sociaux (boycotts, action militante des ONG, etc.). de pressions d'investisseurs se voulant socialement responsables et, last but not least, d'affirmation de valeurs véhiculées par les dirigeants et les employés. La diffusion rapide de ce nouveau référentiel, notamment en Europe, atteste l'efficacité de ce discours auprès des entreprises et des pouvoirs publics.

Il convient évidemment de s'interroger sur l'évidence d'un tel discours et sur sa portée : en quoi est-ce un phénomène nouveau ? quelles sont les filiations, continuités et ruptures entre des pratiques, des théories et des démarches d'entreprise actuelles et d'autres plus anciennes dans le domaine social et environnemental ? à quelles conditions une responsabilité sociale des entreprises est-elle susceptible de s'exercer dans les grandes entreprises ? Telles sont les principales questions que ce numéro se propose d'approfondir.

\section{LES RACINES HISTORIQUES DU DÉVELOPPEMENT DURABLE : CONCEPTIONS ET PRATIQUES}

Les articles ici réunis cherchent à mettre en perspective historique cinq problématiques contemporaines liées au phénomène du développement durable : l'élaboration de l'agenda stratégique des entreprises, la gestion de la contestabilité sociale dans le cadre de projets locaux, l'évolution des formes de régulation conjointe entre l'entreprise et la société. le développement durable comme champ d'innovation, la question des outils de gestion et de l'organisation du développement durable dans les entreprises.

(19) Voir F. Aggeri et alii, Organiser le développement durable, op. cit., chapitre 2 : J. Elkington, Cammibal with forks. The triple bottom line of the 2I" century business, Oxford. Capstone Publishing, 1997.

(20) Cf. WBCSD. The business case for sustainable developmem. Conches, WBCSD. 2002.

(21) Voir D. Vogel. The market for virtue.... op. cit. 


\section{Ruptures et continuités : les évolutions de l'agenda stratégique des entreprises}

La question de l'évolution de l'agenda stratégique des entreprises, en particulier des continuités et des ruptures entre le projet managérial du développement durable et des projets plus anciens concernant la gestion de l'environnement ou bien la gestion sociétale des communautés locales, constitue un premier thème pour des recherches historiques.

Cette question a été abordée par le débat qui a réuni Bertrand Collomb, président de Lafarge et du WBCSD, Richard Armand, ancien délégué général d'Entreprises Pour l'Environnement, Jacqueline Aloisi de Larderel, ancienne directrice de la direction des Technologies du Programme des Nations Unies pour l'Environnement, Michel Capron et Éric Pezet, enseignants-chercheurs de sciences de gestion, Geneviève Massard-Guilbaud, historienne. Les participants ont identifié une première rupture à la fin des années 1960 où la gestion de l'environnement s'institutionnalise dans les grandes entreprises comme objet de gestion légitime, de façon concomitante à la formation de cette notion aux plans scientifique et politique. Ce point est confirmé par l'article de Daniel Boullet qui fait remonter aux années 1960 l'émergence en France de nouvelles figures d'acteurs en charge de la gestion de l'environnement dans les grandes entreprises industrielles. Une deuxième rupture, estiment les participants, se produit dans les années 1990, avec l'émergence du projet managérial du développement durable dont la problématisation plus stratégique est liée à l'émergence de problématiques planétaires (problèmes globaux d'environnement, internationalisation des entreprises et des marchés).

\section{Stratégies de légitimation et gestion de la contestabilité sociale}

La concrétisation du projet managérial du développement durable au plan local (sites, projets industriels) est traitée de manière centrale dans deux articles qui mettent en évidence à la fois l'ancienneté de formes de gestion de la contestabilité et l'évolution des types de réponse apportées par les entreprises.

Dans leur article. François Labelle et Jean Pasquero étudient les évolutions de la conception de la RSE de l'usine d'aluminium d'Alcan située au bord du lac Saint-Jean au Québec. A partir d'une série de portraits depuis les années 1920 jusqu'à nos jours, ils analysent la gestion des relations de l'entreprise avec les communautés locales environnantes. La conception initiale du paternalisme où l'entreprise s'occupe de tout, fait place dans les années 1960 et 1970 à une série de contestations sociales qui, dans les années 2000 , conduisent l'entreprise à construire une approche partenariale qui coïncide avec la formalisation d'une politique de développement durable à l'échelle du groupe. Celle-ci vise à anticiper les contestations sociales possibles en associant les parties concernées à l'action de l'entreprise. Dans cette dernière période la RSE se construit comme une convention négociée entre parties plutôt que comme un devoir moral ou une obligation légale. Les auteurs rejoignent, sur ce point, d'autres analyses récentes qui étudient les formes contemporaines de gestion des relations avec les parties prenantes ${ }^{122 !}$.

(22) J. Andriof. S. Waddock, B. Husted et S. Sutherland Rahman. Unfolding stakeholder thinking, vol. I et II. Sheffield. UK, Greenleaf Publishing. 2002. 
L'article de Marie-Claure Loison et d'Anne Pezet étudie un projet de déversement de boues rouges d'alumine en Méditerranée que l'entreprise Pechiney a mis en ouvre dans les années 1960. Inspiré par une conception confinée de la responsabilité environnementale, ce projet s'est confronté à une importante contestation sociale avant d'aboutir à travers la mobilisation de différents alliés et d'arguments scientifiques, politiques et juridiques. Ce cas constitue, aux yeux des auteurs, une forme primitive, non aboutie, de RSE. Entre volonté d'anticipation et contraintes, plaçant une confiance excessive dans l'autorité de la science et celle de l'Etat, l'entreprise n'est pas alors parvenue à éviter la survenue de fortes controverses publiques avec les différents acteurs sociaux qui se sont mobilisés contre le projet au point, un moment, de rendre incertain son aboutissement.

\section{Des régulations conjointes}

Historiquement, les ajustements des conceptions de l'action des entreprises en matière sociale ou environnementale n'ont pas été conduites d'un bloc, soit de façon autonome soit en pure réaction à des contraintes extérieures. Elles se sont déployées dans le cadre de régulations conjointes ${ }^{1231}$ avec des groupes sociaux et les pouvoirs publics. Les actions individuelles ou collectives des entreprises ont été à la fois orientées et suscitées par des cadres juridiques et réglementaires et par des demandes sociétales qu'ils ont contribué, en retour, à faire évoluer.

Ce processus de régulation conjointe n'est pas nouveau. Dans leur travail historique sur l'émergence du concept de pollution, nouveau "démon moderne ", Christoph Bernhardt et Geneviève Massard-Guilbaud ${ }^{12+1}$ montrent que la pollution émerge comme concept politique dans le courant du XVIIl' siècle et connaît des évolutions successives jusqu au XX" siècle comme produit de l'interaction entre technologies, connaissances scientifiques, culture, valeurs humaines et politiques environnementales. La pollution n'existe au XIX" siècle, rappellent-ils, que s'il y a plainte. Dans son travail de généalogie de l'administration et du droit de l'environnement, Pierre Lascoumes, s'intéressant à l'ancêtre de la loi sur les installations classées - le décret-loi du 15 octobre 1810 sur les installations incommodes et insalubres -, avait mis en évidence que le but du législateur n'était pas alors d'interdire ou de restreindre l'activité des industriels qui s'étaient installés à la périphérie des villes et qui suscitaient, du fait de leurs activités, les réactions des riverains aux nuisances diverses engendrées, mais de réguler les relations de voisinage et de trouver un compromis entre les droits de propriété de l'industriel et ceux des riverains, ce que souligne également G. Massard-Guilbaud dans le débat situé à la fin de ce numéro.

Dans son article, Daniel Boullet met en évidence comment la naissance d'une gestion intentionnelle systématique de l'environnement dans les grandes entreprises industrielles ne les saisit pas à n'importe quel moment de leur histoire mais dans les années 1960, c'est-à-dire au moment où apparaissent de nouvelles formes de régulation publique et où se structure un nouveau type de contestation sociale (le mouvement écologiste). Au départ peu coordonnées

(23) J.-D. Reynaud, Lés règles du jeu : l'action collective ct la régulation socialé. 3x éd.. Paris. Armand Colin, 1997. (24) Voir C. Bernhardt et G. Massard-Guilbaud (dir.). Le démon moderne. La pollution dans les sociétés urbaines ef industrielles d'Europe. Clermont-Ferrand, Presses Universitaires Blaise Pascal, 2004 ; P. Lascoumes, $L$ 'éco-pouvoir. Environnements et politiques. Paris, La Découverte, 1994 ; J.-C. Daumas et P. Mioche, « Histoire des entreprises et environnement : une frontière pour la recherche ", Entreprises ef Histoire. n 35, juin 2004, p. 69-88. 
et ciblées, les actions éparses des entreprises en matière d'environnement s'intègrent ensuite progressivement à la stratégie d'ensemble des entreprises, tandis que se structurent des formes d'action collective (syndicats patronaux, associations d'entreprises, centres techniques) qui visent à diffuser les bonnes pratiques mais également à peser en retour sur les réglementations et sur les normes.

L'article de Yannis Suire permet de mettre en évidence un épisode méconnu de la gestion collective des paysages : la naissance des sociétés de marais, constituées au tournant du XVII siècle pour mettre en valeur le Marais poitevin, où les populations sont alors victimes des inondations et de l'insalubrité. Il montre que cette forme originale de gestion mi-privative mipublique s'est développée grâce aux privilèges et facilités octroyés par les textes royaux.

La question des filiations entre la doctrine du patronage volontaire et les démarches de RSE mises en place par les groupes industriels transnationaux dans les pays en développement est traitée dans l'article de Thierry Hommel. L'auteur souligne deux points communs entre les deux approches : le déploiement de stratégies dans un contexte d'inexistence ou d'effacement relatif de la puissance publique et la construction d'infrastructures pour attirer et fixer une main-d'œuvre qualifiée. L'analyse rejoint, sur ces points, les travaux des historiens sur le paternalisme ${ }^{(29)}$. Il reste cependant que le paternalisme est évidemment loin de faire l'objet d'un consensus parmi les historiens. Ses motifs, doctrines et pratiques ont donné lieu à des interprétations historiques contradictoires à partir des cadres théoriques les plus variés (marxisme, approche foucaldienne, sociologie critique, etc.). Cela rendait d'autant plus nécessaire un travail d'approfondissement historique précis de comparaison entre paternalisme et RSE, ici amorcé.

\section{Le développement durable comme nouveau champ d'innovation}

La question de la construction de capacités d'innovation dans la durée en lien avec des objectifs environnementaux et sociaux ambitieux fait l'objet de l'article d'Hélène Teulon. S'intéressant au cas de Patagonia, figure emblématique de l'entreprise pionnière et militante du développement durable, l'auteur s'interroge sur la capacité d'innovation répétée ${ }^{(26)}$ de l'entreprise depuis trente ans. A contre-courant des préceptes du management, elle montre que Patagonia a su construire un modèle de développement original dont le ressort est un processus d'innovation intensive en matière de développement de produits nouveaux (gamme d'articles de sport) qui lui permet de concilier sa pérennité économique avec des objectifs environnementaux et des valeurs éthiques, revendiquées par le fondateur de l'entreprise. Elle met également en évidence comment l'entreprise a été pionnière dans la minimisation des impacts environnementaux de sa production, en mobilisant l'analyse de cycle de vie ou encore en modifiant ses matières premières (substitution partielle du coton biologique au coton standard), jouant ainsi un rôle d'émulation à l'égard de concurrents qui s'orientent à leur tour dans cette voie. Elle montre enfin la cohérence d'une stratégie qui joue simultanément sur l'organisation, la gouvernance, la gestion des ressources humaines et celle des relations avec les sous-traitants et avec les consommateurs.

(25) Voir P. Lefebvre, L'invention de la grande entreprise. Travail, hiérarchie, marché. Francé, fin XVIItr-début XX' siècle, Paris, PUF, 2003.

(26) Pour une analyse des principes et des pratiques d'une gestion de l'innovation répétée et intensive dans les entreprises, on pourra se référer au livre de P. Le Masson. B. Weil et A. Hatchuel, Les processus d'innovation. Conception innovante et croissance des entreprises. Paris, Hermès-Lavoisier. 2006. 


\section{L'organisation et les outils de gestion du développement durable}

Il n'a pas été possible, dans le cadre de ce numéro, d'aborder toutes les questions contemporaines liées aux pratiques de développement durable des entreprises. C'est le cas de la problématique de l'organisation et des outils de gestion qui n'est que rapidement évoquée dans le débat et dans le compte rendu de la thèse de Jean-Pascal Gond. Ces questions ne sont pas nouvelles mais elles invitent à un réexamen des pratiques contemporaines à l'aune de travaux historiques. On pense en particulier aux travaux américains de la fin des années 1960 développés dans le cadre d'un projet de recherche dénommé Corporate Social Responsiveness, mené à la Harvard Business School sous la houlette de Raymond A. Bauer autour de la question de l'organisation concrète des réponses apportées par les grandes entreprises américaines aux enjeux de RSE. Ces auteurs se sont intéressés, à partir d'enquêtes approfondies menées dans 40 grandes entreprises américaines, à la naissance dans les grandes entreprises américaines de la figure de l'expert en questions sociétales (social issue specialist), au cycle de vie des questions sociétales et aux capacités d'apprentissage de la grande entreprise moderne du point de vue de l'appropriation de la nouveauté, en particulier des outils d'audit social qui voient le jour à cette époque ${ }^{[27}$. Il est d'autant plus utile de réévaluer aujourd'hui ces travaux que ce mouvement de recherche a progressivement disparu de la littérature sur la RSE au profit d'interrogations sur l'éthique, les modèles de relation avec les parties concernées ou l'étude des corrélations entre performance économique et performance financière. Elles sont discutées dans deux ouvrages récents en langue française sur le sujet qui s'interrogent sur la spécificité et les effets propres des instrumentations mises en place dans le domaine de la RSE et du développement durable et en matière de reporting sociétal des entreprises ${ }^{12 \times 1}$. Dans la thèse récente de Jean-Pascal Gond, analysée à la fin de ce numéro par Isabelle Huault, la question de l'institutionnalisation du champ de l'investissement socialement responsable est traitée à travers une analyse longitudinale des dispositifs de «calculabilité » mis en place par l'agence de notation sociétale ARESE au cours des années 1990 en France. Plus généralement, les questions d'organisation et d'instrumentation sont évoquées dans le débat retranscrit dans ce numéro : le développement durable n'est-il pour les entreprises qu'une extension d'outils de management de la qualité ou bien s'accompagne-t-il de démarches spécifiques comme l'éco-conception, l'analyse de cycle de vie ou le reporting ? Les participants ont souligné l'émergence de nouvelles formes d'instrumentation économique ainsi que l'émergence de dispositifs de reporting sociétaux adossés à des référentiels volontaires transnationaux dont il reste à étudier la généalogie et les effets sur les pratiques managériales.

\section{De nouvelles responsabilités pour l'entreprise : vers un nouveau statut de l'entreprise?}

Mettant en évidence les limites, voire la crise des systèmes de régulation en place. les débats sur le développement durable posent des questions inédites sur la responsabilité des

(27) Pour une discussion et une présentation de ces travaux, on pourra se référer à A. Acquier et F. Aggeri, « La responsabilité sociale des entreprises... ". art. cit.

(28) Voir M. Capron et F. Quairel-Lanoizelée. Mvthes et réalités de l'entreprise respomsables. Paris, La Découverte. 2004. et F. Aggeri e't alii. Organiser le développème'm darable'.... op. cit. 
entreprises et sur les modèles de gouvernance auxquels elles sont associées ${ }^{291}$. C'est l'objet de l'article d'Alexia Leseur que de montrer les limites d'une conception de l'entreprise comme simple association d'individus et d'explorer une compréhension de l'entreprise comme entité morale ayant des devoirs et pas seulement des droits. Elle propose, dans cette perspective, une grille de lecture permettant d'analyser la légitimité des comportements des entreprises qui disent s'inscrire dans la perspective du développement durable. La question est intéressante car elle s'inscrit dans une évolution parallèle du droit qui reconnaît désormais la responsabilité pénale de l'entreprise en tant que telle et pas seulement celle de tel ou tel dirigeant. La question fondamentale posée est celle de l'affirmation du statut moral d'entités collectives diverses, au nombre desquelles on trouve l'entreprise. Les entreprises sont couramment exposées à des jugements moraux et leurs dirigeants acceptent de façon croissante de se situer sur ce terrain et de revendiquer une responsabilité morale. Si tel était le cas, c'est certainement toute la théorie de l'entreprise qui devrait être reconsidérée pour préciser à quels devoirs les entreprises sont légitimement soumises et à quels droits elles peuvent légitimement prétendre. Pour la prise en charge de la thématique du développement durable, il y a là un enjeu important.

(29) A propos de la modélisation de l'entreprise comme projet collectif incluant la création de nouveaux potentiels d'action, on pourra consulter A. Hatchuel el B. Segrestin, "La société contre l'entreprise : vers une nouvelle norme d'entreprise à progrès collectif », Droit êt Société (à paraître). 


\section{ONT COLLABORÉ À CE NUMÉRO}

Franck Aggeri, ancien élève de l'ENS Cachan, maître-assistant de sciences de gestion à l'Ecole des Mines de Paris. Membre du conseil stratégique du programme Eco-technologies et développement durable de l'Agence Nationale pour la Recherche. Article récent : "Les régimes de gouvernementalité dans le domaine de l'environnement ", in A. Hatchuel, É. Pezet, K. Starkey, O. Lenay (dir.), Gouvernement, organisation et gestion : I'héritage de Michel Foucault, Sainte-Foy, Presses Universitaires de Laval, 2005.

Jacqueline Aloisi de Larderel, ancienne directrice de la Division Technologie, Industrie et Economie du Programme des Nations Unies pour l'Environnement. Après avoir exercé, entre 1972 et 1986, diverses responsabilités au ministère chargé de l'Environnement (chargée de mission, chef du Service des Déchets, adjoint au directeur de la Prévention des Pollutions et des Nuisances), elle a été de 1987 à 2003 au Programme des Nations Unies pour l'Environnement, en tant que directrice de la Division Technologie. Industrie et Economie. En 2001, elle est également nommée sous-directeur exécutif du PNUE. Depuis 2003, elle est membre d'un certain nombre de conseils d'administration ou de groupes de conseil mis en place par diverses organisations ou ONG : Fondation Ensemble. ICLEI (Local Governments for Sustainability). E\&Co (Energy for Development), Global Reporting Initiative, ADEME... Toutes ces activités l'ont amenée à développer et diriger diverses recherches sur les thèmes liés à la problématique industrie et développement durable. En particulier, elle a dirigé la préparation, pour le sommet de Johannesburg en 2002, de rapports d'évaluation de la performance de différents secteurs industriels.

Richard Armand, après avoir exercé la fonction de Délégué général de l'association Entreprises pour l'Environnement (EpE) de 1998 à 2005, se focalise désormais sur la lutte contre le changement climatique à moyen et long termes, plus précisément après 2012. Actuellement conseiller de l'association. Ses recherches sont soit internes à EpE, soit reprises dans des documents mis en ligne sur le site interne de l'association.

Xavier Boivin, chargé de recherche à l'INRA en éthologie (Unité de Recherche sur les Herbivores de Clermont-Ferrand-Theix), travaille sur les déterminants du développement des relations homme-ongulés d'élevage. Publication : X. Boivin, P. Le Neindre, A. Boissy, J. Lensink, G. Trillat, I. Veissier, « Eleveur et grands herbivores : une relation à entretenir ", INRA Prod. Anim., 16 (2), 2003. p. 101-115.

Daniel Boullet, agrégé d'histoire et docteur en histoire de l'Université Paris X-Nanterre. étudie l'histoire de l'industrie française sous l'angle des questions environnementales depuis 1950. Livre : Entreprises et enironnement en France de 1960 à 1990 : les chemins d'une prise de conscience, Genève, Librairie Droz. 2006.

Michel Capron, professeur de sciences de gestion à l'Université Paris VIII - Saint-Denis, dirige une spécialité de master « Conseil en organisation et gestion des innovations sociales " et à l'Université Paris XII - Val de Marne une spécialité de master « Management de la responsabilité sociale des entreprises ». Président du Conseil d'orientation du Réseau International de Recherche sur les Organisations et le Développement Durable (RIODD), il est également l'un des animateurs du Forum citoyen pour la responsabilité sociale des entreprises. II a consacré l'essentiel de ses recherches de ces dernières années à la responsabilité sociale des entreprises et au développement durable. Livres récents : (en collaboration avec Françoise Quairel) Mythes et réalités de l'entreprise responsable. Paris, La Découverte, 2004. et La responsabilité sociale d'entreprise. Paris, La Découverte, 2007. 
Bertrand Collomb. Président de Lafarge, Membre de l'Institut (Académie des Sciences Morales et Politiques). A créé en 1972 le Centre de Recherche en Gestion de l'Ecole Polytechnique. A présidé depuis sa création en 1992 jusqu'en 2001 l'Association Nationale pour la Valorisation Interdisciplinaire des Sciences Sociales dans les Entreprises (ANVIE). Intéressé par le développement durable depuis le sommet de Rio, il a été un des membres fondateurs du World Business Council for Sustainable Development, un groupe de 200 entreprises mondiales impliquées dans le développement durable, dont il a été président en 2004 et 2005 . A fait de nombreuses conférences ou articles sur le développement durable. A contribué à plusieurs reprises à Entreprises et Histoire.

Olivier Godard, directeur de recherche au CNRS, Laboratoire d'économétrie de l'Ecole Polytechnique, professeur à l'Ecole Polytechnique. Président du Conseil scientifique Agriculture et développement durable de l'Agence Nationale pour la Recherche. Dernier livre : La question de la précaution en milieu professionnel, Paris, EDP Sciences, 2006.

Ivan Grinberg est secrétaire général de l'Institut pour l'histoire de l'aluminium (www.histalu.org). Il a notamment publié L'aluminium. un si léger métal. Paris, Gallimard, Découvertes, 2003 (prix Roberval 2004) et Pomblière, fabrique de métaux depuis 1898. Grenoble. Presses Universitaires de Grenoble. 1998.

François Hochereau, sociologue, chargé de recherche à l'INRA (département Sciences pour l'Action et le Développement). Après s'être intéressé aux dynamiques d'apprentissage organisationnel autour des outils informatiques, il s'intéresse aujourd 'hui au rôle de la mesure comme « opérateur social », en tant que résultat et support cognitif de processus d'acquisition de connaissances, de construction de jugements et de prises de décisions collectives. Ses travaux se centrent sur l'introduction de nouveaux critères de sélection et de production en agriculture. Article récent : «Concrétisation et normalisation : les in- jonctions contradictoires de l'inscription de l'informatique dans l'organisation ", Réseaux, $\mathrm{n}^{\circ} 135-136,2006$, p. 288-321.

Thierry Hommel est chargé de mission à la chaire de développement durable de l'Institut d'Etudes Politiques de Paris et chercheur associé à l'Iddri. Institut du développment durable et des relations internationales. et membre du COPE (comité opérationnel d'éthique) du CNRS depuis 2004. Ses travaux portent principalement sur la gestion stratégique de la réputation des entreprises. Il a publié récemment Stratégie des firmes industrielles et contestation sociales, Paris, INRA Editions, 2004, et, avec Olivier Godard, "Les multinationales, un enjeu stratégique pour le développement durable ? ", Revue internationale et stratégique, hiver 2005-2006.

François Labelle. docteur en administration à Montréal UQAM, HEC, Université Concordia, Université McGill (2005), professeur de management et de stratégie de gestion à l'université du Québec à Trois-Rivières. Articles récents : «Les 3 « $C$ » de la performance sociétale organisationnelle (PSO) : convention, compromis, cohérence ". Gestion, été 2006 (dossier spécial sur la Responsabilité sociale des entreprises) : "Vers une reformulation du concept de responsabilité sociale en termes de convention sociale $»$. Actes du colloque Advancing theory in CSR : an intercontinental dialogue, Montréal CSR International Workshop 2006. UQAM. Chaire de responsabilité sociale et de développement durable, 12-15 octobre.

Alexia Leseur, ingénieure, docteure en économie de l'environnement de l'Ecole Polytechnique (thèse sur l'équité dans l'allocation initiale des permis d’émission négociables entre entreprises). Alliant analyse philosophique et économique, elle a notamment développé l'idée d'un statut moral de l'entreprise et ses implications.

Marie-Claire Loison, ancienne élève de l'ENS Cachan, agrégée d'économie-gestion, Attachée Temporaire d'Enseignement et de Recherche à l'Université Paris-Dauphine, prépare son doctorat en sciences de gestion au 